\title{
Fatal varicella pneumonia in an unvaccinated child with Down Syndrome: a case report
}

\author{
Diletta Valentini ${ }^{{ }^{*}}$, Simona Bianchi ${ }^{1}$, Chiara Di Camillo $^{1}$, Anna Chiara Vittucci ${ }^{1}$, Michaela Veronika Gonfiantini ${ }^{1}$, \\ Rita De Vito ${ }^{2}$ and Alberto Villani ${ }^{1}$
}

\begin{abstract}
Background: Varicella is an acute infectious disease common during childhood. It has mostly an uncomplicated course in early childhood. Neverthless, it may result in severe complications, especially in particular age groups and clinical conditions. Down Syndrome represents a risk factor for developing complications, because of the frequent comorbidities and their immunodeficiency.

Case presentation: A 2-year-old white Caucasian female affected by Down Syndrome was referred to our hospital for cardiac arrest in course of varicella disease. After cardiopulmonary resuscitation and stabilization, her clinical conditions didn't improve and she developed a massive pulmonary hemorrage, which led her to exitus.

Conclusions: Mortality due to varicella infection is rare, but it is more common in subjects with immune deficit or chronic pathologies, and in particular age-groups. The importance of the vaccine for preventable infectious diseases is stressed in this paper, in which we present a case of death in an unvaccinated cardiopathic child with Down Syndrome affected by varicella.
\end{abstract}

Keywords: Down Syndrome, Immunodeficiency, Varicella, Pneumonia, Vaccination, Case report

\section{Background}

Varicella is an acute, exanthematous, highly infectious disease, that most commonly occurs in childhood. Varicella normally has a benign course, but can occasionally develop into a more serious illness, especially in adults, immunodeficient children, pregnant women, newborn babies [1]. A lethal outcome is very rare, with a mortality rate fluctuating between 0.29 and 0.46 deaths per 1 million. The introduction of the vaccine in 1995 has substantially decreased varicella incidence, hospitalizations, and deaths [2].

The most common complications of varicella are bacterial skin infection, sepsis, pneumonia, and central nervous system events such as cerebellar ataxia and encephalitis $[3,4]$.

\footnotetext{
* Correspondence: diletta.valentini@opbg.net

${ }^{1}$ Pediatric and Infectious Disease Unit, Bambino Gesù Children's Hospital,

IRCCS, Rome, Italy

Full list of author information is available at the end of the article
}

\section{Case presentation}

A 2-years-old white Caucasian female affected by Down Syndrome (DS) and surgically corrected at 3 months of age for a subaortic intraventricular defect (IVD), with a history of 2 episodes of pneumonia, was admitted in the emergency room of our hospital due to a cardiac arrest during her varicella illness. She had never been vaccinated up to the moment, that's why she contracted the disease from her sister. The night before her admission to the hospital, she began to manifest episodes of hypotonia associated to periods of crying. At 4 o'clock she began to show signs of a generalized hypotonia and she was taken to our hospital by her parents, where she arrived in cardiac arrest. After Cardio-Pulmonary Resuscitation (CPR-PALS) her spontaneous breathing was restored. The clinical course was characterized by complete areflexia, with bilateral mydriasis. Breathing pattern was characterized by ARDS that required high frequency mechanical ventilation and Nitric Oxide with transient improvement. 
We proceeded to perform a chest X-ray (CXR), which revealed multiple foci of parenchymal spread to both lungs and pleural effusion obliterating share of the breast-phrenic cost (Fig. 1). An abdominal ultrasound showed the presence of abdominal effusion in all quadrants and laboratory tests revealed the presence of IgM antibodies against varicella, positive PCR for varicella antigen, absence of bacterial infections (coltures of blood and urine), prolonged PT and PTT, and altered D-Dimer. Tests for immunological functions were performed (Table 1).

The next day, the respiratory condition didn't improve and a new CXR showed an impairment of the spread, and a massive pulmonary hemorrhage. In the absence of recovery of the main indicators of organ perfusion, she was declared dead.

At macroscopic examination the lungs were heavy, firm and plum-colored, with diffuse areas of hemorrhage and necrosis.

Histologically there are interstitial pneumonitis, diffuse necrosis and hemorrhage in the pulmonary parenchyma (Fig. 2).

\section{Conclusions}

The estimated global burden of disease-specific mortality caused by varicella is considerably lower than that due to other major infectious diseases such as measles, pertussis, rotavirus, or invasive pneumococcal disease. Based on conservative estimates, the global annual varicella disease burden reports 4.2 million severe complications leading to hospitalization and 4200 deaths [5].

The prevalence of immunocompromising conditions including HIV infection and the kind of treatment available, are factors which influence the course of the disease. In healthy children, varicella is usually selflimiting and benign [6].

Groups at higher risk for severe complications are: neonates, infants, pregnant women, adults, and immunocompromised patients.

The type of varicella complications depends on the patient's age. A study of Gowin et al. demonstrates that the average age of varicella-complications in hospitalized children is 3.12 years $[7,8]$. The youngest children had pneumonia, and the oldest meningitis/meningoencephalitis $[9,10]$.

Older children considered to be more susceptible to antibody-mediated inflammatory reactions, whereas younger, less immunocompetent patients are more frequently affected by bacterial suprainfections of the skin or of the respiratory tract $[11,12]$.

Respiratory tract infections were present in younger children, and usually developed at the beginning of the varicella infection [7]. The high frequency of respiratory tract complications reflects the biology of the virus. The virus enters the host through the respiratory tract and then spreads in the bloodstream. A cytopathic effect of

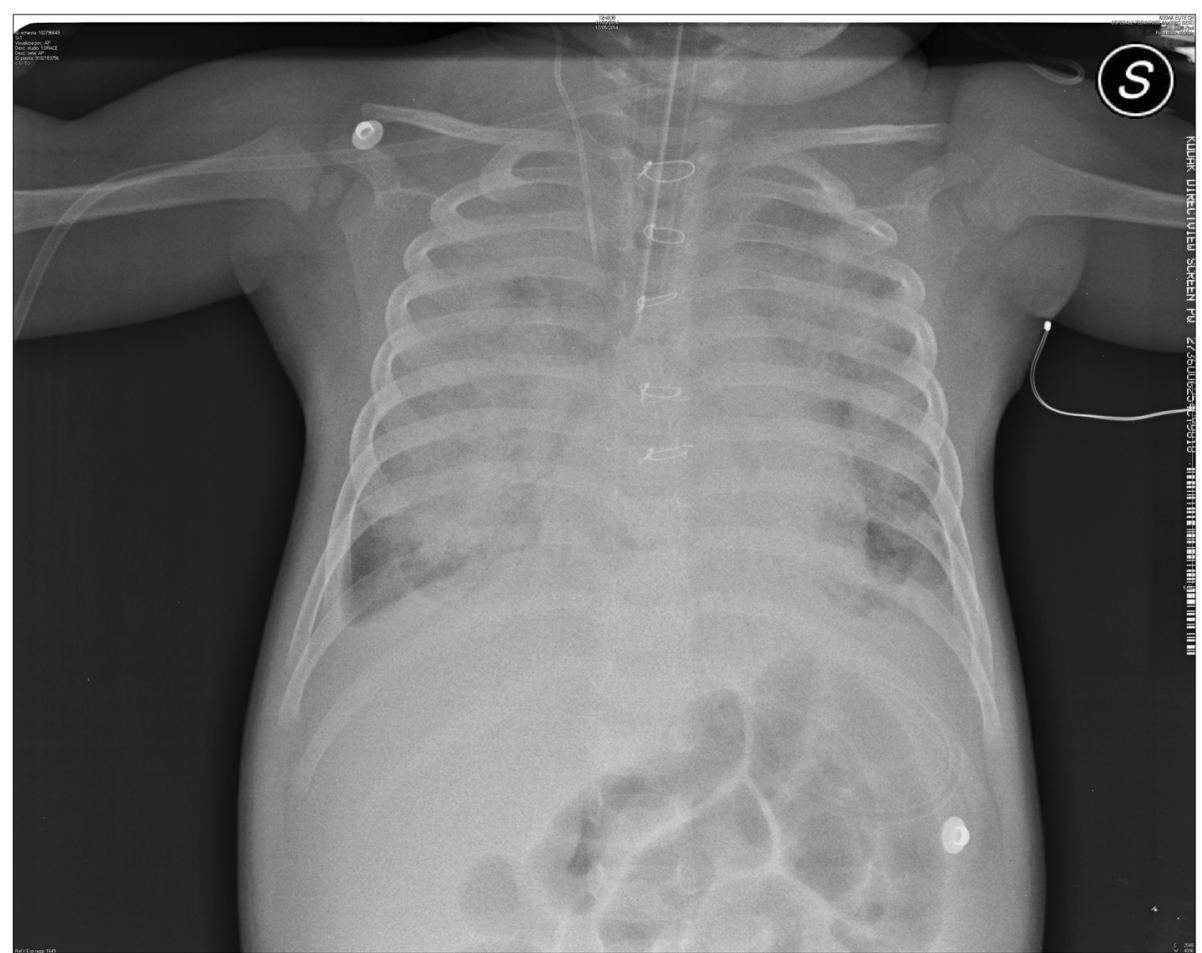

Fig. 1 Chest X-Ray: multiple foci of parenchymal spread to both lungs and pleural effusion obliterating share of the breast-phrenic cost 
Table 1 Blood investigations performed at the emergency room access

\begin{tabular}{lll}
\hline Investigation & Value & Reference value \\
\hline White bllod cells & $15.65\left({ }^{*} 103 / \mathrm{uL}\right)$ & $5.5-15$ \\
Red blood cells & $4.03\left({ }^{*} 106 /{ }^{*} \mathrm{uL}\right)$ & $3.6-5$ \\
Hemoglobin & $9(\mathrm{~g} / \mathrm{dL})$ & $10.5-15.5$ \\
Platelets & $131(103 / \mathrm{uL})$ & $150-450$ \\
PTT-s & $50.5($ seconds) & $25-34$ \\
PTT-r & $1.73($ seconds) & $0.85-1.15$ \\
Trombin Time & $27.6($ seconds) & $16-22$ \\
Antitrombine III & $34(\%)$ & $75-120$ \\
Fibrinogen Dimeri & $6.7(\mathrm{microg} / \mathrm{mL})$ & $<0.5$ \\
LDH & $4150(\mathrm{UI} / \mathrm{L})$ & $230-470$ \\
CPK & $3595(\mathrm{UI} / \mathrm{L})$ & $32-211$ \\
CD3-pan T & $56.4(\%)$ & $58-75$ \\
CD4 T Helper & $16.8(\%)$ & $29-47$ \\
CD8 T Suppressor/Cytotoxic & $39.7(\%)$ & $17-33$ \\
CD19 Pan B & $36(\%)$ & $14-30$ \\
CD16 + CD56+ & $6.7(\%)$ & $4-17$ \\
VZV PCR & positive & Negative \\
VZV IgG & negative & - \\
VZV IgM & positive & - \\
\hline
\end{tabular}

the varicella virus on the alveolar epithelium causes pneumonia. Desquamated alveolar cells contribute to reduce gas exchange.

Hematological complications are observed in $1-2 \%$ of children with varicella. Patients usually remain asymptomatic. Like many other thrombocytopenias and anemias during viral infections, those in patients with varicella are transient and require no treatment. Laboratory tests are not performed routinely in patients with varicella [13].

Our case suffered from acute respiratory distress syndrome (ARDS) caused by varicella, and associated to hematological disorders that provoked the development of pulmonary hemorrhage, which caused death.

Children who are diagnosed with Down syndrome and who have comorbidities such as immunodeficiency or cardiopathy have high rates of viral and bacterial infections such as influenza and pneumococcal infections [14]. The most common symptoms reported in children with DS are infections of the respiratory tract suggesting a B-cell defect [15]. Diseases related to T-cell deficiency, such as infection with intra-cellular microorganism, fungi and opportunistic pathogens are rare [16].

Varicella is one of the most infective disease that affects the pediatric population.

Guidelines stress the importance of an anti-varicella vaccination for all children older than 12 months as well as children with DS [17]. The only contraindication of the vaccine is severe immunodeficiency of the $\mathrm{T}$ cells [6]. Despite the fact that most children hospitalized with varicella complications were immunologically healthy, risks are superior in subjects with chronic conditions $[6,18]$.

Children with DS have a higher risk of being hospitalized for viral respiractory tract infections, even in the absence of coexisting risk factors [19], and the mortality rate in severe ill DS children admitted for medical reasons is high and is predominantly associated with respiratory conditions [20]. As the burden of preventable infections in children diagnosed with chronic diseases is high in terms

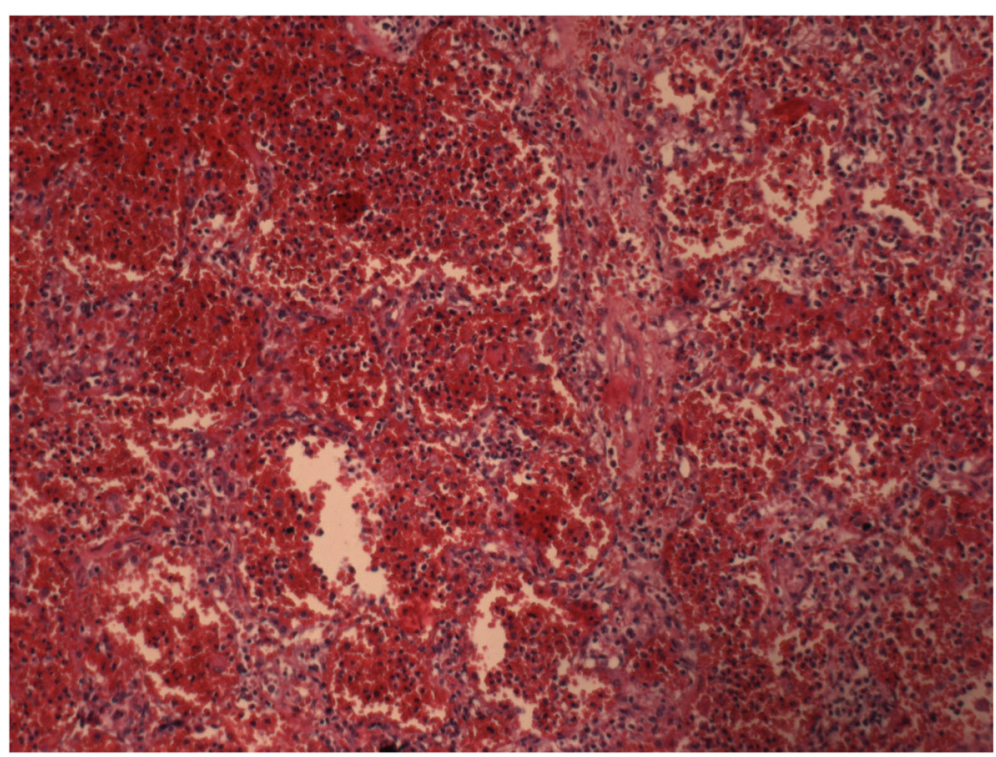

Fig. 2 Histological image that showed interstitial pneumonitis and diffuse necrosis and hemorrhage in the pulmonary parenchyma 
of incidence and severity, it is essential to protect these children with timely administration of vaccinations according to the current recommendations.

Even among populations at risk, varicella vaccine is the less used. Although Italian Down children have the best vaccination coverage among all patients with chronic disease [21], it is clear that it is desirable to improve vaccination coverage among risk groups and also among the healthy population.

Implemented interventions, with special reminders to parents and general practitioners, are necessary to promote timely vaccination in DS children who are susceptible to preventable infections and prone to severe complications.

This case underlines how varicella may lead to severe, potentially life-threatening complications in unvaccinated children and adolescents, and may demonstrate too the benefits of varicella vaccination.

\section{Abbreviations}

ARDS: Acute respiratory distress syndrome; CPR-PALS: Cardio-Pulmonary Resuscitation; CXR: Chest X-ray; DS: Down Syndrome

\section{Aknowledgements}

No aknowledgements.

\section{Funding}

Diletta Valentini, who wrote the first draft of the manuscript, wasn't given by anyone any grant or other form of payment to produce the manuscript.

\section{Availability of data and materials}

The dataset supporting the conclusions of this article is available in the Bambino Gesù Children Hospital repository.

\section{Authors' contributions}

DV wrote the manuscript. SB collected the clinical and laboratory data, and wrote the manuscript. CDC collected the bibliography. AV reviewed the immunization schedule for Down Syndrome, in order to write the discussion. MVG reviewed the clinical complications of varicella, in order to write the discussion. RDV performed the pathological analysis. AV reviewed the manuscript. All authors read and approved the final manuscript.

\section{Competing interests}

The authors declare that they have no competing interests.

\section{Consent for publication}

Written informed consent was obtained from the patient's parents/legal guardians for publication of this case report and any accompanying images. A copy of the written consent is available for the review by the Editor-in-Chief of this journal.

\section{Ethics approval and consent to participate}

Not applicable.

\section{Author details}

'Pediatric and Infectious Disease Unit, Bambino Gesù Children's Hospital, IRCCS, Rome, Italy. ${ }^{2}$ Pathology Unit, Bambino Gesù Children's Hospital, IRCCS, Rome, Italy.

Received: 7 August 2016 Accepted: 11 November 2016

Published online: 17 November 2016

\section{References}

1. Kimberlin D, Brady MT, Jackson MA, Long SS. Red Book, 30th ed, American Academy of Pediatrics. 2015:846-60.
2. Huong Q, Nguyen MPH, Aisha O. Decline in mortality due to varicella after implementation of varicella vaccination in the United States. N Engl J Med. 2005;352:450-8.

3. Losurdo G, Bertoluzzo L, Canale F, Timitilli A, Bondi E, Castagnola E, Giacchino R. Varicella and its complications as cause of hospitalization. Infez Med. 2005:13:229-34.

4. Bozzola E, Bozzola M, Tozzi AE, Calcaterra V, Longo D, Kryzystofiak A, Villani A. Acute cerebellitis in varicella: a ten years case series and systematic review of the literature. It J Pediatr. 2014;40:57.

5. Trucchi C, Gabutti G, Rota MC, Bella A. Burden of varicella in Italy, 20012010: analysis of data from multiple sources and assessment of universal vaccination impact in three pilot regions. J Med Microbiol. 2015;64:1387-94.

6. World Health Organisation. Varicella and herpes zoster vaccines: WHO position paper 2014;9:265-288

7. Dubos F, Grandbastien B, Hue V, Martinot A. Epidemiology of hospital admissions for paediatric varicella infections: a one-year prospective survey in the pre-vaccine era. Epidemiol Infect. 2007;135:131-8.

8. Liese JG, Grote V, Rosenfeld E, Fischer R, Belohradsky BH, Kries RV. The burden of varicella complications before the introduction of routine varicella vaccination in Germany. Pediatr Infect Dis J. 2008;27:119-24.

9. Gowin E, Wysocki J, Michalak M. Don't forget how severe varicella can be-complications of varicella in children in a defined Polish population. Int J Infect Dis. 2013;17:e485-9.

10. Bozzola E, Tozzi AE, Bozzola M, Krzysztofiak A, Valentini D, Grandin A, Villani A. Neurological complications of varicella in childhood: case series and a systematic review of the literature. Vaccine. 2012;30:5785-90.

11. Hervas D, Henales V, Yeste S, Figuerola J, Hervas J. How frequent is varicella associated pneumonia in children? Eur J Clin Microbiol Infect Dis. 2011;30:435-7.

12. Patel RA, Binns HJ, Shulman ST. Reduction in pediatric hospitalizations for varicella-related invasive group A streptococcal infections in the varicella vaccine era. Pediatrics. 2004;144:68-74.

13. Bozzola E, Quondamcarlo A, Krzysztofiak A, Pandolfi E, Lancella L, Tozzi AE. Haematological complications in otherwise healthy children hospitalized for varicella. Vaccine. 2011:29:1534-7.

14. American Academy of Pediatrics. Committee on Infectious Diseases. Policy statement: recommendation for the prevention of pneumococcal infections, including the use of pneumococcal conjugate vaccine (Prevenar), pneumococcal polysaccharide vaccine, and antibiotic prophylaxis. Pediatrics. 2000;106:362-6.

15. Carsetti R, Valentini D, Marcellini V, Scarsella M, Marasco E, Giustini F, Bartuli A, Villani A, Ugazio AG. Reduced numbers of switched memory B cells with high terminal differentiation potential in Down syndrome. Eur J Immunol. 2015;45:903-14.

16. Verstegen RH, Kusters MA, Gemen EF, de Vries E. Down Syndrome BLymphocyte subpopulations, intrinsic defect or decresead T-lymphocyte help. Pediatr Res. 2010;67:563-9.

17. Gallo G, Mel R, Rota MC. Guide to contraindications to vaccination. 2009, v, 92 p. Rapporti ISTISAN 09/13 (in Italian).

18. Oxman MN, Schmader KE. Editorial commentary: zoster vaccine in immunocompromised patients: time to reconsider current recommendations. Clin Infect Dis. 2014:59:920-2.

19. Zachariah P, Ruttenber M, Simões EA. Down syndrome and hospitalizations due to respiratory syncytial virus: a population-based study. J Pediatr. 2012:160:827-31.

20. Joffre C, Lesage F, Bustarret O, Hubert P, Oualha M. Children with Down syndrome: Clinical course and mortality-associated factors in a French medical paediatric intensive care unit. J Paediatr Child Health. 2016;52:595-9.

21. Pandolfi E, Carlonia E, Marinoa MG, Ciofi degli Atti ML, Gesualdo F, Romano $M$, et al. Immunization coverage and timeliness of vaccination in Italian children with chronic diseases. Vaccine. 2012;30:5172-8. 